\title{
The Influence of Gender Grouping on Female Students' Academic Engage- ment and Achievement in Engineering and Biology: A Case of Small Group Work in Design-based Learning (Work in Progress)
}

\section{Miancheng Guo, University of Massachusetts}

Miancheng Guo is a PhD candidate in Science Education at the University of Massachusetts Amherst.

Dr. Martina Nieswandt, University of Massachusetts

Martina Nieswandt is an Associate Professor of Science Education and Associate Dean for Research and Engagement in the College of Education at the University of Massachusetts, Amherst. Her research focuses on the relationship between motivation, affects and learning associated with K-16 science concepts and various instructional contexts (e.g., small groups, project-based learning) utilizing mixed-methods approaches.

\section{Dr. Elizabeth McEneaney, University of Massachusetts}

Dr. McEneaney is Associate Professor in the Department of Teacher Education and Curriculum Studies at the University of Massachusetts - Amherst. She is a former high school mathematics and science teacher, and earned a Ph.D. in Sociology from Stanford University. An associate editor for the Journal of Curriculum Studies, she has research interests in equity and access to STEM Education, and the influence of globalization on STEM curricula. 


\section{The Influence of Gender Grouping on Female Students' Academic Engagement and Achievement in Engineering and Biology: A Case of Small Group Work in Design-Based Learning (Work in Progress)}

\section{Introduction}

During the past 30 years, much attention has been drawn to the lack of women in STEM fields and the need to attract and retain them in these fields. In the relevant literature, the influence of gender grouping on variables such as female students' interest, self-efficacy, participation/engagement and achievement in STEM subjects has been a salient line of research. However, researchers have arrived at mixed findings. Also, while researchers have investigated the influence of gender grouping on female students in various instructional contexts, such as authentic engineering design tasks and collaborative learning in inquirybased science, little has been done on design-based science (DBS), a relatively new pedagogy in which students construct scientific and engineering knowledge and problem-solving skills through designing components, artifacts or systems (Fortus, Krajcik, Dershimer, Marx, \& Mamlok - Naaman, 2005). This paper is an effort to address these inadequacies.

For this purpose, the present study explored (1) the relationship between gender grouping and female students' behavioral/emotional/cognitive engagement in DBS learning; and (2) the relationship between gender grouping and female students' achievement in engineering practices and biology concepts. The specific context of this study was a DBS task in a high school biology class in which 3- or 4-member student groups designed an oil spill cleanup system. For the purpose of our study, we systematically varied the gender composition of the groups so there were $100 \%, 75 \%, 50 \%, 33 \%$, and $25 \%$ female groups and $100 \%$ male groups.

\section{Literature Review}

To inform our study, we conducted a comprehensive literature review regarding gender grouping at different levels (e.g., small group, classroom, school) in different instructional approaches (e.g., inquiry-based learning, paper-and-pencil problem solving) in various fields (e.g., science, engineering). The following is a succinct summary of our findings.

Gender grouping has been studied in various fields, such as science, mathematics, and engineering. In science, it has been studied in formal and informal settings with more conventional pedagogies, such as group discussion and inquiry-based science (e.g., Estrada, 2007) as well as with the relatively new pedagogy - DBS (e.g., Watson \& Lyons, 2009). Various formats of student activities have been used in these studies, such as paper-andpencil problem solving (e.g., Harskamp, et al., 2005), computer-aided virtual scientific inquiry (e.g., Kemp, 2005), real-life hands-on scientific inquiry (e.g., Estrada, 2007) and engineering design (e.g., Gnesdilow, et al., 2013), or exposure to authentic lab research experiences (e.g., Hirsch, et al., 2014). The term "group" has been used widely in these studies and referred to different units, including pairs (e.g., Harskamp, et al., 2008), small groups (e.g., Gnesdilow et al., 2013), classes (e.g., Friend, 2006), programs (e.g., Richardson et al., 2003), or even camps (e.g., Hughes, et al., 2013). Methods-wise, some researchers simply compared single-gender and mixed-gender units (e.g., Friend, 2006); and a few others systematically varied the gender composition of student units (e.g., 25\%, 50\%, 75\% ... female; Dasgupta et al., 2015). Also, importantly, these studies have measured a wide variety of variables, such as student identity (e.g., Hughes et al., 2013), attitudes (e.g., Watson \& Lyons, 2009), competence (e.g., Häussler \& Hoffmann, 2002), interest (e.g., Hughes et al., 2013), self-efficacy (e.g., Dasgupta et al., 2016), engagement (e.g., Bennett et al., 2010), career aspiration (e.g., Dasgupta et al., 2016), and achievement (e.g., Baker, 2005).

All these studies have produced mixed findings. While some researchers found gender grouping to be related to improved levels of girls' attitudes, interest, engagement, and achievement (e.g., Harskamp et al., 2008;), others could not establish such correlations (e.g., 
Kemp, 2005; ) or even revealed reversed relationships (e.g., Gnesdilow et al., 2013). This phenomenon is consistent with what other researchers have noticed (e.g., Friend, 2006). Although small group work has some great advantages, only a relatively small number of studies on gender grouping in STEM fields have been conducted at the small group level. Given this situation and given that gender grouping research in DBS is sparse (Gnesdilow et al., 2013), it can be safely inferred that gender grouping research in DBS at the small group level is even more sparse. Actually, this has been our impression during our ongoing and persistent review of literature.

Another important phenomenon is that, while a certain amount of research investigated the relationship between gender grouping and student engagement, much of it adopted a unidimensional measure of engagement (Wang \& Eccles, 2011), and few dissected this concept into its behavioral, emotional and cognitive dimensions (Fredricks, et al.,2004). Yet, there is still one more issue. During our extensive search for and review of gender grouping literature, we noticed that only a small number of studies focused on student engagement in engineering design (e.g., Dasgupta et al., 2015; Watson \& Lyons, 2009; Hirsch et al., 2014; Gnesdilow et al., 2013), and none explored student engagement in the different steps of the engineering design cycle. However, with the release of Next Generation of Science Standards (NGSS), which outline engineering practices as part of science learning and gives engineering design the same status as scientific inquiry (NGSS Lead States, 2013), it is important to further study student engagement (and learning) during engineering design.

In summary, based on the literature we conclude that there may be no gender grouping studies which explored the following characteristics simultaneously:

- Gender grouping's influences on girls' engagement at the small group level in DBS;

- Variation of student groups' gender composition (i.e., 25\%, 50\% female, and so on);

- A 3-dimensional perspective of engagement - behavioral, emotional, and cognitive;

- Focus on students'3D engagement in each step of the engineering design process, and

- Students achievement in both science and engineering.

Apparently, this is a hole in the literature and it is important to address it. This study makes such an effort, by investigating the above-mentioned relationships.

\section{Conceptual Framework}

The conceptual framework of this study includes multiple components, but due to space limit here we only include the concept of the engineering design process. For this concept, we used the definition provided by the 2016 Massachusetts Science and Technology/Engineering Curriculum Framework (Massachusetts Department of Elementary and Secondary Education, 2016): Researching the Problem - Conceptual Design - Embodiment Design - Test and Refine.

\section{Participants}

\section{Methods}

A total of 185 students (51 three- or four- member groups) participated in this study. These students were from nine classes in four high schools in Massachusetts and Vermont. Given that we are still analyzing the large amount of video data and the space of this paper is limited, here we only present the student engagement data of the following four groups: Group 4-four girls (100\% female); Group 3-three girls and one boy (75\% female); Group 2-two girls and two boys (50\% female); Group 1-one girl and two boys (33\% female).

All 185 students were originally arranged into four- or three- member groups by the researchers according to their levels of interest in biology (so that each group was a mixture of different levels of interest in biology), but their teachers made necessary adjustments to 
avoid students who wouldn't work well together being in the same group. In this study, for our research purposes, we picked the above four groups of different gender compositions. Before this DBS task, these students had worked in these groups on a scientific inquiry task.

\section{Data Collection and Analysis}

Qualitative data were collected by videotaping each student group's whole work process. Quantitative data were collected by pre- and post-activity surveys regarding students' interest, competency, skills and knowledge in engineering and biology.

Sequence analysis (qualitative data converted to quantitative information). Since our purpose was to investigate whether the varying gender composition of student groups would influence girls' group-level engagement in their DBS activity, we analyzed data at the gender-specific subgroup level. That is, our unit of analysis was the subgroup.

Different engineering design steps may require different behavioral, emotional and cognitive engagement. For example, conceptual design is considered the most cognitively intensive in the engineering design process (Kim, 2011). Therefore, throughout the whole design process, students may have engaged in their task differently, behaviorally emotionally and cognitively. Thus, we perceive the videos recording their design processes as temporal data. In order to analyze such data, we used sequence analysis - a temporal data analysis method (Abbott, 1995). Each video was divided into a number of two-minute segments for adequate coding, and each segment was watched and compared with predetermined indicators that reflect the three types of engagement and thus record the presence of each type of engagement at the sub-group level. Due to space limit of this paper, these indicators will have to be omitted. But here it is important to report that they were developed by the authors of the present paper and have been validated for a very similar purpose in a previous study (Guo, Nieswandt, McEneaney \& Howe, 2016).

Based on a comparison between each two-minute video segment and these indicators, students' behavioral, emotional and cognitive engagement in this short time period could be identified. In each two-minute video segment, if all/majority/half/minority/none of the students in a subgroup showed a sign of behavioral engagement for over 60 seconds, then we labeled this segment as all/majority/half/minority/none behaviorally engaged for that subgroup. For emotional and cognitive engagement, each segment was labeled the same way, but the threshold time period was set to be 30 seconds. As the two-minute value of the video segments, these threshold values were also set arbitrarily, but based on our repeated experiments. For behavioral engagement, we decided that 60 seconds was a reasonable threshold value because the behavioral engagement indicators we used represented relatively long-lasting behaviors/actions and if a student engaged in such behaviors/actions for only 30 or 50 seconds in a two-minute time period, we considered it inadequate. For emotional and cognitive engagement, the videos recorded a considerable number of instances in which student showed clear signs of engagement for less than 60 seconds (e.g., excitement about their successful model testing, drawing a picture of their design, etc.), thus, we decided 30 seconds was a reasonable threshold value for not missing important signs.

To record each subgroup's engagement level (i.e., all/majority/half/minority/none engaged) in a segment, we assigned a numerical value to each level, as shown in Table 1 . All the numerical recordings were then inputted into $\mathrm{R}$ (a statistical software), which then produced visual representations (i.e., sequences) of each subgroup's three types of engagement. During this process, within $\mathrm{R}$, we assigned a color to each level of engagement so that these different levels can be visualized by different colors in the sequences. Table 1 reports these color codes. 
Elaborated running records (ERRs) (qualitative data analysis). We used this method to qualitatively capture the interactions among group members and other salient phenomena that were related to their behavioral/emotional/cognitive engagement. First, we developed an ERRs table which contained three columns: Time, Running Record, and Reviewer Comments. Then, we watched each video throughout, identified each event/phenomenon that was a manifestation of or related to students' behavioral/emotional/cognitive engagement based on a pre-determined indicator system, and recorded it in the Running Record column. Each event/phenomenon was recorded by narrative description with master indicators inserted wherever necessary in the passage. Here, a master indicator is a single letter put in a square bracket denoting a certain type of engagement. For example, cognitive engagement is denoted by " $[\mathrm{C}]$ " and behavioral engagement by " $[\mathrm{B}]$ ". In a passage describing an event/phenomenon, if a master indicator is inserted somewhere, then it means the part of this passage that's before this master indicator describes a phenomenon that's a manifestation of the type of engagement denoted by this master indicator. In the Time column, the period in which an event/phenomenon happened was recorded. In the Reviewer Comments column, we put in any materials that we thought were helpful for understanding the contents in the Running Record column, such as notes and screenshots from the video.

Quantitative data analysis. To explore the relationship between gender grouping and students' achievement, we used SPSS to calculate Pearson correlation coefficients. Here, the

Table 1 Different engagement levels and their numerical values and color codes

\begin{tabular}{|l|c|c|}
\hline \multicolumn{1}{|c|}{ Engagement level } & Numerical Value & Color Code \\
\hline All engaged & 5 & Dark green \\
\hline Majority engaged & 4 & Light green \\
\hline Half engaged & 3 & Yellow \\
\hline Minority engaged & 2 & Purple \\
\hline None engaged & 1 & Red \\
\hline
\end{tabular}

independent variable was the percentage of girls in the group, and the dependent variables were students' test scores in biology knowledge and engineering practices.

\section{Results and Discussion}

Results of sequence analysis of students' behavioral engagement are shown in Figure 1 and 2 (due to space limit, visual results for emotional and cognitive engagement will have to be omitted; instead, we will narratively report these results). As can be seen from these figures, a rough pattern of change of the students' levels of behavioral engagement can be observed from Group 1 to 4, with the increase of the percentage of girls in the group, the female students' overall levels of behavioral engagement increased and the male students' decreased. Also, within the female subgroups, there is another rough pattern - in Female Subgroups 3 and 4 , where the percentage of girls in the group was higher than $50 \%$, the girls' levels of behavioral engagement maintained high throughout the whole design process, while in Female Subgroups 1 and 2, where the percentage of girls in the group was lower than 50\%, the girls' levels of behavioral engagement were much lower.

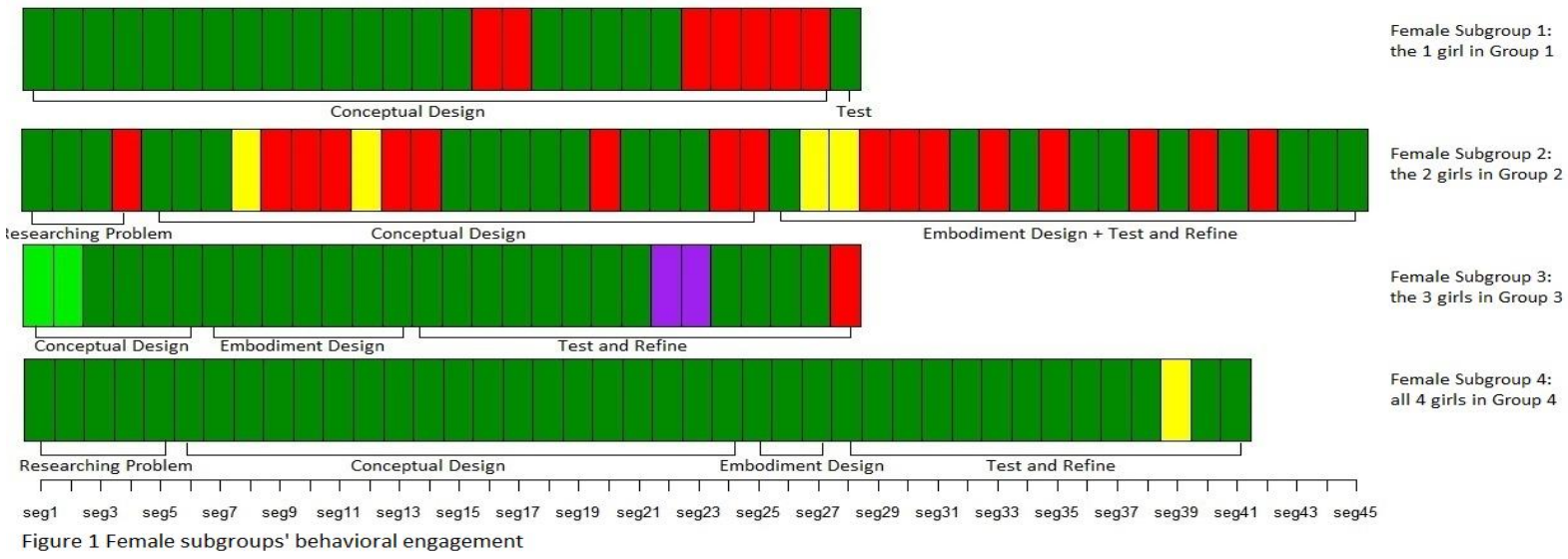




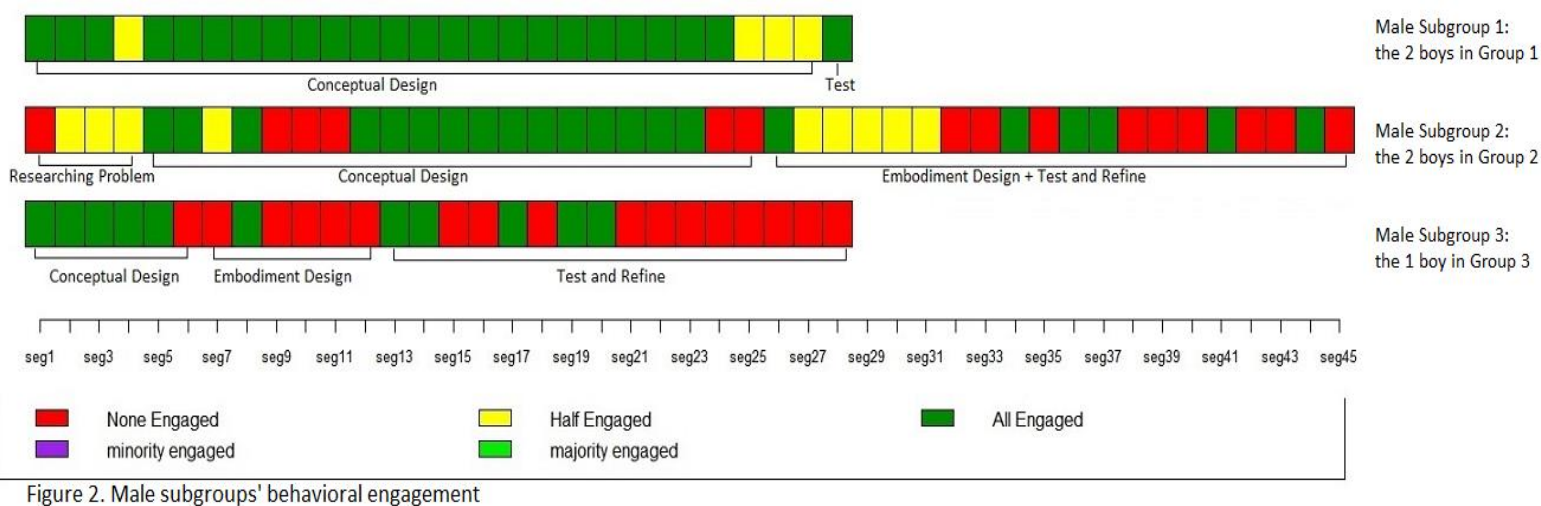

For emotional and cognitive engagement, similar patterns also exist. While it is not very clear that the girls' overall levels of emotional and cognitive engagement increased linearly from Group 1 to 4 (with the increase of group female percentage), it is obvious that in Groups 3 and 4 , where there were more than $50 \%$ of girls in the group, these girls' levels of these two types of engagement were considerably higher than girls in Groups 1 and 2, where the group female percentage was 50\% or lower. In addition, it's also clearly visible that in Groups 3 and 4 , the girls maintained high levels of emotional and cognitive engagement throughout their whole engineering design process.

Why was all this? In Groups 1 and 2 where girls were less than 50\%, were their low levels of behavioral, emotional and cognitive engagement related to their male peers or some other factors? Dasgupta's (2011) stereotype inoculation model proposes that in an achievement context contact with ingroup peers functions as a "social vaccine" that inoculates individuals against stereotype-based self-doubt and thus enhance individuals' positive attitudes toward the achievement domain, their identification with it, their self-efficacy, and their motivation to pursue achievement goals in the domain and that such contact is particularly important for those whose ingroup is a minority and negatively stereotyped (in this case, girls in STEM fields). Does this theory apply here? We looked into our ERRs and did find a lot of qualitative descriptions of positive interactions among female students in the same group in Groups 3 and 4 (75\% and 100\% girls, respectively). In Group 2 where there were two girls and two boys, although in the ERRs we found descriptions of positive interactions between the two girls, we also found descriptions of how the girls were ignored or prevented by the boys while they wanted to participate/contribute. This is also true for Group 1. Dasgupta (2011) introduces that generally in an achievement context the critical mass for a minority group to perceive themselves well and do well is $33 \%$, but it seems that in our study this number is $50 \%$, and this is consistent with a previous study of the authors of this paper (Guo et al., 2016).

Quantitative analyses revealed both non-significant and significant correlations between the percentage of girls in the group and students' achievement. There was no significant correlation between group female percentage and female students' achievement in biology knowledge $(\mathrm{r}=0.124, \mathrm{n}=99, p=0.222)$ or in engineering practice $(\mathrm{r}=-0.11, \mathrm{n}=99, p=0.914)$. However, there was a significant correlation between group female percentage and the achievement in biology knowledge of all students (both girls and boys) ( $\mathrm{r}=0.162, \mathrm{n}=185$, $p=0.027$ ), although there was no significant correlation between group female percentage and all students' achievement in engineering practice $(\mathrm{r}=0.088, \mathrm{n}=185, p=0.233)$.

How to interpret all these achievement results in relation to the female and male students' levels of engagement? Would they be more influenced by behavioral, emotional, or cognitive engagement? What were the specific factors that were related to the students' changing levels of engagement? We will continue with our data analysis with these questions in mind and report more results when they become available. 


\section{Acknowledgement}

This study is part of NSF-funded study named Project REESE (DRL-1252339). We are grateful for the support of NSF. Also, we would like to thank Dr. Susannah Howe of Smith College for her input on our understanding of the engineering design process.

References:

Abbott, A. (1995). Sequence Analysis: New Methods for Old Ideas. Annual Review of Sociology. http://doi.org/10.1146/annurev.so.21.080195.000521

Baker, D. (2002). Good intentions: An experiment in middle school single-sex science and mathematics classrooms with high minority enrolment. Journal of Women and Minorities in Science and Engineering, 8, 1-23.

Bennett, J., Hogarth, S., Lubben, F., Campbell, B., \& Robinson, A. (2010). Talking Science: The research evidence on the use of small group discussions in science teaching. International Journal of Science Education.

doi:10.1080/09500690802713507

Connell, J. P. (1990). Context, self, and action: A motivational analysis of self-system processes across the life-span. In D. Cicchetti (Ed.), The self in transition: Infancy to childhood (pp. 61-97). Chicago: University of Chicago Press.

Dasgupta, N. (2011). Ingroup Experts and Peers as Social Vaccines Who Inoculate the Self-Concept: The Stereotype Inoculation Model. Psychological Inquiry. http://doi.org/10.1080/1047840X.2011.607313

Estrada, E. (2007). The effects of inquiry and single-gender grouping on second grade girls' attitudes and participation in science. University of Central Florida Electronic Theses and Dissertations. Retrieved from http://digital.library.ucf.edu/cdm/ref/collection/ETD/id/3705

Fortus, D., Krajcik, J., Dershimer, R. C., Marx, R. W., \& Mamlok-Naaman, R. (2005). Design-based science and real-world problem-solving. International Journal of Science Education. doi:10.1080/09500690500038165

Fredricks, J. A., Blumenfeld, P. C., \& Paris, A. H. (2004). School Engagement: Potential of the Concept, State of the Evidence. Review of Educational Research. doi:10.3102/00346543074001059

Friend, J. (2006). Research on Same-Gender Grouping in Eighth Grade Science Classrooms. Research in Middle Level Education Online, 30, 1-15. Retrieved from

http://search.ebscohost.com/login.aspx?direct=true\&AuthType=ip,cookie,url,uid\&db=ehh\&AN=32831958\&site=ehost-live

Gnesdilow, D., Evenstone, A., Rutledge, J., Sullivan, S. \& Puntambekar, S. (2013). Group Work in the Science Classroom: How Gender Composition May Affect Individual Performance. Paper presented at the 2013 Computer Supported Collaborative Learning Conference, University of Wisconsin-Madison

Guo, M., Nieswandt, M., McEneaney, E. H. \& Howe, S.V. (2016, April). Students' engagement in different steps of the engineering design process in a design-based biology activity. Paper presented at the Annual International Conference of the National Association for Research in Science Teaching, Baltimore, MD.

Harskamp, E., Ding, N. \& Suhre, C. (2008). Group Composition and Its Effect on Female and Male Problem-Solving in Science Education. Educational Research, 50, 307-318. http://doi.org/10.1080/00131880802499688

Häussler, P., \& Hoffmann, L. (2002). An intervention study to enhance girls' interest, self-concept, and achievement in physics classes. Journal of Research in Science Teaching, 39(9), 870-888. http://doi.org/10.1002/tea.10048

Hirsch, L. S., Berliner-Heyman, S., Cano, R., Carpinelli, J. \& Kimmel, H., (June 2014). The effects of single vs. mixed gender engineering enrichment programs on elementary students' perceptions of engineers. Paper presented at the $121^{\text {st }}$ American Society for Engineering Education Conference and Exposition, Indianapolis, IN

Hughes, R. M., Nzekwe, B., \& Molyneaux, K. J. (2013). The Single Sex Debate for Girls in Science: A Comparison Between Two Informal Science Programs on Middle School Students' STEM Identity Formation. Research in Science Education, 43(5), 1979-2007. http://doi.org/10.1007/s11165-012-9345-7

Kemp, R.L. (2005). The impact of gender-specific and mixed-gender cooperative groups on female gifted students using computer-assisted, problem-based learning. A thesis submitted to the Graduate School, Valdosta State University. In partial fulfillment of requirements for the degree of Education Specialist in Instructional Technology in the Department of Curriculum and Instructional Technology of the College of Education. Retrieved from http://chiron.valdosta.edu/are/vol4no1/pdf/kemprarticle.pdf

Kim, J. (2011). Modeling cognitive and affective processes of designers in the early stages of design: mental categorization of information processing. Other. Arts et Metiers ParisTech, 2011. English. <NNT: 2011ENAM0011>. <pastel-00594203>

Massachusetts Department of Elementary and Secondary Education (2016). 2016 Massachusetts Science and Technology/Engineering Curriculum Framework. Retrieved at: http://www.doe.mass.edu/frameworks/scitech/2016-04.pdf NGSS Lead States. (2013). Next Generation Science Standards. Achieve, Inc.

Richardson, G., Hammrich, P., \& Livingston, B. (2003). Improving elementary school girls' attitudes, perception, and achievement in science and mathematics: Hindsights and new visions of the sisters in science program as an equity reform model. Journal of Women \& Minorities in Science \& Engineering, 9 (3/4), 333- 348.

Wang, M. Te, \& Eccles, J. S. (2012). Adolescent Behavioral, Emotional, and Cognitive Engagement Trajectories in School and Their Differential Relations to Educational Success. Journal of Research on Adolescence, 22(1), 31-39. https://doi.org/10.1111/j.1532-7795.2011.00753.x

Watson, J.\& Lyons,J. (2009). Teaching engineering in single-gender middle-school classrooms. Paper presented at ASEE Annual Conference and Exposition, Austin, Texas, June 2009. 